\title{
Estudio geoarqueológico de palimpsestos en las dunas de la localidad Sayape (San Luis, Argentina)
}

\section{(1) Daniela Storchi Lobos*}

\author{
Fecha de defensa: 6 de marzo de 2018 \\ Directora: Dra. Karen Borrazzo \\ Co-director: Dr. Guillermo Heider \\ Jurados: Dres. Luis Borrero y Roberto Kokot
}

\section{Introducción}

El registro arqueológico del centro-sur de la provincia de San Luis suele encontrarse en superficie formando palimpsestos entre dunas (Greslebin, 1924, 1928; Heider, 2015). Ello se debe a la dinámica eólica imperante en este sector (Forman et al., 2014; Iriondo y Kröhling, 1995) que genera hoyadas de deflación en donde materiales arqueológicos y naturales se acumulan y superponen. En estos contextos, propiedades básicas del registro arqueológico tales como resolución e integridad se ven considerablemente afectadas, lo que ha sido tradicionalmente señalado como una limitación para el estudio de este tipo de conjuntos (ver Fanning et al., 2009). Hasta el momento, las investigaciones llevadas adelante en esta región por Heider (2015) han permitido anclar cronológicamente algunas ocupaciones utilizando artefactos diagnósticos. Así, este autor ha propuesto ocupaciones en momentos tempranos (ca.800o años AP) a partir del hallazgo de puntas de proyectil tipo Ayampitín, y otras durante el Holoceno tardío identificadas por la presencia de puntas triangulares pequeñas y tecnología cerámica (Heider, 2015). Como puede observarse, la posibilidad de establecer cronologías en este sector de la provincia ha quedado restringida al hallazgo de materiales diagnósticos, siendo en apariencia dificultoso ubicar cronológicamente aquellos conjuntos de superficie que carecen de ellos (que suelen ser los más frecuentes). Una posible respuesta a este problema se logra con el estudio de los contextos en los que los materiales se encuentran depositados desde un abordaje geoarqueológico, es decir, incluyendo en el análisis arqueológico y cronológico a las geoformas que contienen los conjuntos, la matriz que los alberga y, finalmente, el ambiente y sus cambios como transformadores del registro arqueológico (Favier Dubois, 2009). Este abordaje para comprender la problemática de los palimpsestos se ve enriquecido mediante estudios tafonómicos que permiten revelar parte de la historia postdepositacional a partir del estudio de las modificaciones exhibidas por los materiales (Behrensmeyer, 1978; Schiffer,

\footnotetext{
* Investigaciones Arqueológicas y Paleontológicas del Cuaternario Pampeano (INCUAPA), Facultad de Ciencias Sociales, Universidad Nacional del Centro de la Provincia de Buenos Aires (UNICEN) CONICET. Av. Del Valle 5737 (CP B740oJWI) Olavarría, Buenos Aires, Argentina.E-mail: dsl.Isd.12@gmail.com
}

1987, entre otros). En conjunto, estas aproximaciones proveen valiosos datos sobre esos materiales que, a simple vista, parecen tan limitados en su potencial informativo.

\section{Área de estudio}

Los campos de dunas del centro-sur de San Luis forman parte del Sistema Eólico Pampeano (Iriondo y Kröhling, 1995; Zárate y Tripaldi, 2012). Los depósitos sedimentarios que lo conforman desde el Pleistoceno tardío son arenas finas bien seleccionadas, que en conjunto evidencian condiciones áridas a semiáridas desde aquellos momentos (Forman et al., 2014; Zárate y Tripaldi, 2012). Las geoformas más conspicuas son las dunas parabólicas (en general vegetadas) y los bajos de deflación, los cuales suelen presentar lagunas en su interior (Iriondo y Kröhling, 1995; Zárate y Tripaldi, 2012). Los depósitos eólicos presentan diferentes unidades estratigráficas (sedimentarias y pedológicas) que responden a distintos momentos en la historia de formación del paisaje y entre ellas se evidencian eventos de erosión (Forman et al., 2014). De esta manera, en algunos casos se registran discordancias con hiatos temporales que abarcan la totalidad del Holoceno, mientras que en otros casos se preservan unidades que representan diferentes momentos de este interglaciar. El principal evento morfogenético registrado para el Holoceno tuvo lugar hacia principios del siglo XX (década de 1930) durante una severa sequía asociada con un déficit en las precipitaciones y la profunda modificación antrópica del paisaje por la agricultura (Forman et al., 2014).

En este contexto geomorfológico fue definida un área de estudio acotada denominada localidad Sayape. Ésta se encuentra a $24 \mathrm{~km}$ al SSO de la ciudad de Villa Mercedes, comprende un área de $100 \mathrm{~km}^{2}$ y obtiene su nombre por incluir la laguna homónima (Figura 1). En esta localidad se han detectado materiales arqueológicos ubicados en dunas cercanas a lagunas (Greslebin, 1924, 1928; Heider, 2015). Una de las características particulares que posee el área es la carencia de fuentes de materias primas líticas en las inmediaciones (Heider, 2015). Los conjuntos arqueológicos recuperados en la localidad Sayape se presentaban en superficie, identificándose materiales líticos (principalmente lascas y microlascas, siendo escasos los instrumentos formales), restos óseos (con diferentes grados de fragmentación, meteorización y termoalteración) y cerámica (Figura 2) (Greslebin, 1924, 1928; Heider, 2015). En asociación con estos materiales arqueológicos suelen hallarse rizoconcreciones (Heider, 2015), las cuales atestiguan la presencia de suelos/paleosuelos ahora 


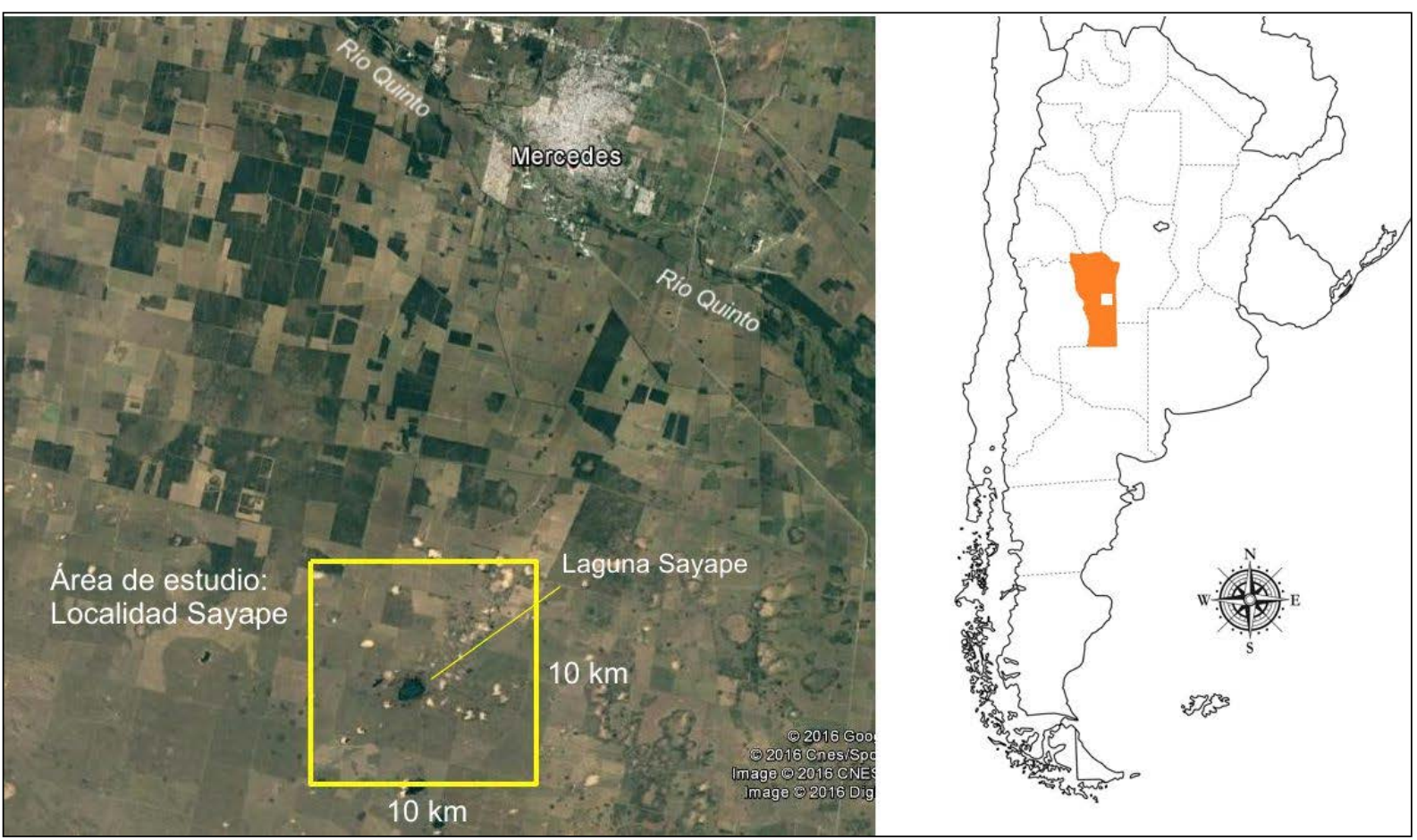

Figura 1. Ubicación del área de estudio y de su principal laguna (izq. imagen Google Earth 2017).

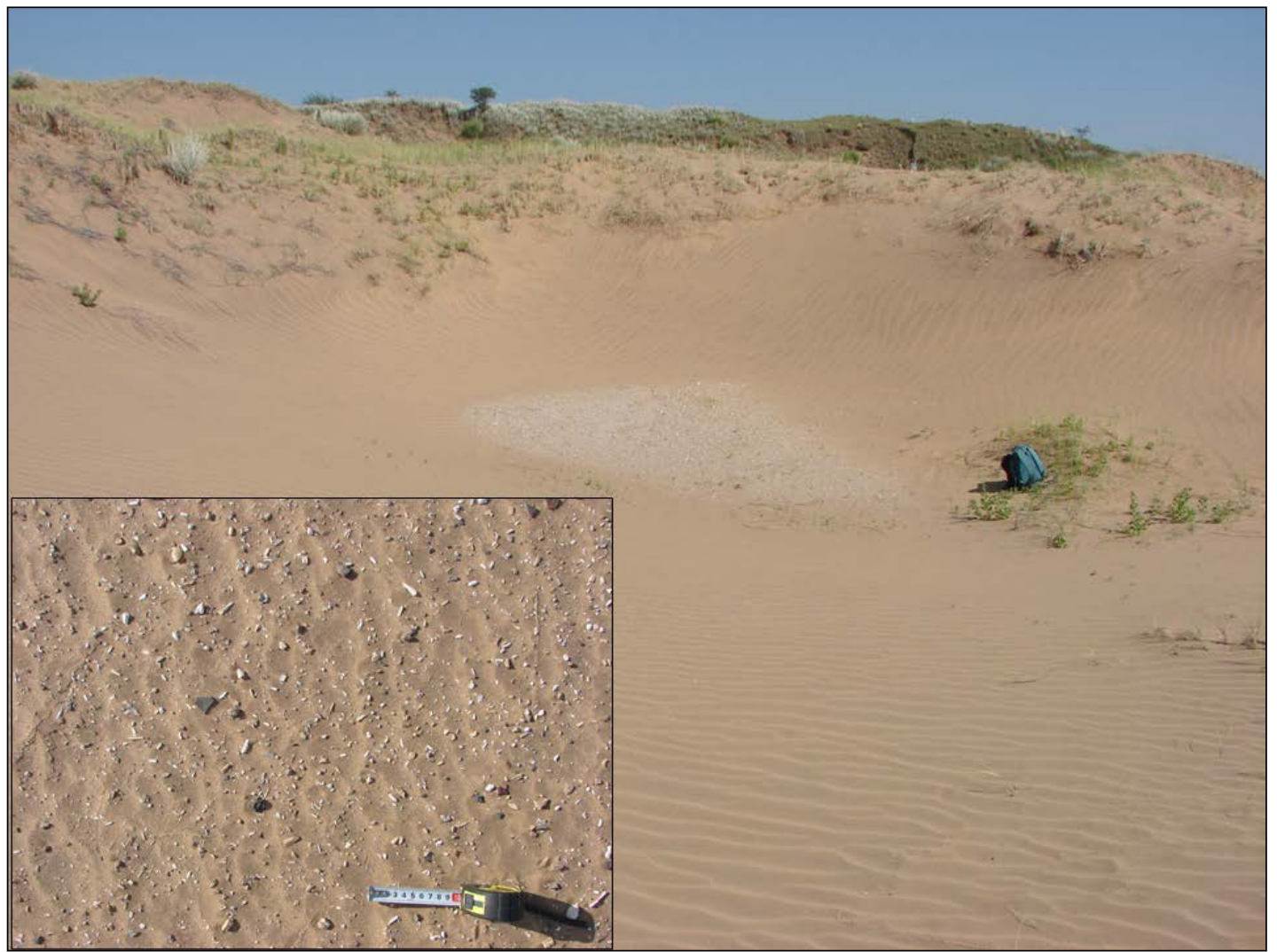

Figura 2. Vista de los materiales en la superficie de una hoyada de deflación. El recuadro (izq.) muestra un acercamiento a los materiales y pueden observarse líticos, huesos y cerámicas muy fragmentados. 
erosionados. En este contexto los principales agentes naturales intervinientes en la formación de los conjuntos de superficie son el viento (Heider, 2015) y la gravedad (reptaje), mientras que la visibilidad se restringe sustancialmente a aquellos espacios en los que actuó la deflación (hoyadas).

\section{Marco teórico}

Esta investigación se enmarcó en la Arqueología Contextual propuesta por Butzer (1989), que considera de fundamental importancia el estudio de la ecología humana en un ambiente que es dinámico y no homogéneo. Este enfoque teórico entiende que el ambiente es un factor importante en el análisis del contexto arqueológico ya que es allí donde las evidencias materiales de las decisiones y acciones antrópicas son depositadas, coincidiendo con la propuesta de la Arqueología Ambiental con respecto a que tanto ese registro cultural como el natural se amalgaman durante la historia del paisaje y forman parte de él, necesitando el arqueólogo descifrar ambos para realizar inferencias sobre el pasado. Teniendo estas consideraciones en cuenta, lo más relevante para la Arqueología Contextual es el análisis de los sitios arqueológicos y su ubicación en el ambiente (tanto pasado como presente), en lugar de estudiar sólo los artefactos arqueológicos en sí mismos (Butzer, 1989). Para abordar estas investigaciones se recurre fundamentalmente a la geoarqueología, entendida como la investigación arqueológica que utiliza los métodos y conceptos de las ciencias de la Tierra para responder preguntas arqueológicas (Butzer, 1989; Waters, 1992). El alcance de la investigación geoarqueológica abarca no sólo la formación inicial de los sitios y sus potenciales cambios durante la ocupación, sino también su sepultamiento y erosión parcial posteriores y la eventual dispersión y mezcla de artefactos y otros restos materiales en los sitios o fuera de ellos (Butzer, 1989).

\section{Objetivos}

Esta investigación tuvo por objetivo general proporcionar herramientas provenientes de la geoarqueología y la tafonomía que permitan obtener información cronológica y contextual de los palimpsestos sin necesidad de recurrir exclusivamente a los materiales diagnósticos. Los objetivos específicos fueron: a) evaluar cuáles son los agentes responsables de la presencia de materiales en las hoyadas de deflación, b) lograr un acercamiento a la historia tafonómica de los mismos para ayudar a comprender los procesos postdepositacionales actuantes, y c) evaluar los cambios en el paisaje y su relación con el registro arqueológico. La geoarqueología y la tafonomía permiten comprender en forma más acabada los contextos de depositación, exposición y alteración de los materiales en este ambiente dominado por la dinámica eólica.

\section{Metodología}

Con el propósito de obtener mayor información cronológica, contextual y tafonómica de los conjuntos de superficie en este ambiente, se seleccionaron cuatro sectores de prospección dentro de la localidad Sayape a partir del reconocimiento, comprensión y evaluación de la geología y geomorfología del área (sectores A, B, C y D). Se escogieron tres hoyadas de deflación con materiales arqueológicos del sector $\mathrm{A}$ denominadas $\mathrm{H}_{3}, \mathrm{H}_{5}$ y $\mathrm{H}_{6}$ para realizar muestreos sistemáticos de un metro cuadrado en los fondos de esos rasgos erosivos. En esas unidades de muestreo se recuperaron todos los materiales presentes en la capa superficial empleando un tamiz con malla de $2 \mathrm{~mm}$. Se tomaron muestras de sedimentos en $\mathrm{H}_{3}, 30 \mathrm{~cm}$ por debajo de los materiales de superficie, para realizar dataciones por OSL (Optically Stimulated Luminiscence) y análisis sedimentológicos (granulometría, porcentaje de materia orgánica, $\mathrm{pH}$ y contenido de carbonatos).

A la muestra de materiales generada en estas campañas se sumó otra de una colección que se encuentra en INCUAPA (CONICET-UNICEN), efectuada unos años antes en este sector de la provincia.

Los materiales líticos, óseos y cerámicos fueron analizados desde una perspectiva tafonómica considerando variables específicas para cada tipo de material (Behrensmeyer, 1978; Borrazzo, 2006; Fernández Jalvo y Andrews, 2016; Ozán y Berón, 2016).

Siguiendo la propuesta de Colman y colaboradores (1987) se utilizaron tres métodos para obtener cronologías en estos contextos, con cinco indicadores: 1) el método radiogénico, en este caso OSL, que permite obtener edades numéricas; 2) el método geomorfológico, que incluye 2a) el grado de desarrollo de los perfiles de suelos y 2 b) las modificaciones progresivas del paisaje, proporcionando edades relativas entre geoformas; y 3 ) el método de correlación, que incluye 3a) las correlaciones estratigráficas y 3b) la presencia de fósiles y artefactos diagnósticos presentes en las unidades del paisaje proporcionando asimismo relaciones temporales entre los conjuntos.

\section{Resultados}

El total de materiales analizados fue de 7.181. Los artefactos líticos fueron 637, de los que se analizaron 133 que tenían tamaño mayor a $10 \mathrm{~mm}$. En dicha muestra se observó que el $15,79 \%$ presentaba carbonatos en su superficie mientras que el 42,86\% mostraba coloración por óxidos de hierro. El $66,92 \%$ presentaban abrasión, siendo el estado 1 el más representado (51,88\%). Los materiales óseos fueron 6.443 en total y registraron un alto índice de fragmentación. En cuanto a los estados predominó el "meteorizado" seguido 
por el "quemado". Sólo dos fragmentos óseos mostraron huellas de corte mientras que 17 presentaron marcas de raíces en sus superficies, aparentemente generadas luego de un proceso de combustión. Los materiales cerámicos analizados fueron 101 en total y los resultados obtenidos muestran que el grado de meteorización predominante (sensu Ozán y Berón, 2016) fue el 1 (55\%) seguido del grado $2(22 \%)$ y luego el grado $3(12 \%)$. En esos fragmentos altamente meteorizados y abradidos fue posible observar que entre los antiplásticos empleados predominaron granos de cuarzo, calcedonias, micas y fragmentos de rocas máficas, todos de tamaño sábulo (entre $2 \mathrm{~mm}$ y $4 \mathrm{~mm}$ ). Los análisis sedimentológicos indicaron que la matriz eólica está compuesta predominantemente por arenas finas muy bien seleccionadas y representa un ambiente oxidante, de buen drenaje (dado por la textura) y alcalino dominado por los carbonatos, que generan un $\mathrm{pH}$ algo mayor a 8 . El fechado por OSL arrojó una edad en el Pleistoceno tardío final que indica que los materiales apoyan sobre una superficie pleistocena, es decir que todas las posibles ocupaciones del Holoceno se encuentran resumidas en una única superficie.

\section{Conclusión}

La investigación enfocada en la geoarqueología y la tafonomía de los palimpsestos en superficie permitió observar algunos aspectos importantes de los procesos de formación locales del registro. Entre ellos, los agentes responsables de la ocurrencia de los materiales en estas hoyadas de deflación, la historia tafonómica de los mismos, la evaluación de los cambios en el paisaje y su relación con los materiales arqueológicos, además de comenzar a conocer cuánto tiempo resumen los palimpsestos aquí estudiados. De esta manera fue posible establecer que: 1) muchos de los materiales se encontraron enterrados bajo uno o más suelos antes de su exposición y tuvieron contacto con las napas freáticas; 2 ) durante ciertos lapsos del Holoceno las condiciones ambientales locales fueron estables, con erosión y agradación nula o casi nula, y una disponibilidad de agua diferente a la actual; 3) la mayoría de los materiales líticos y cerámicos evidencian poca abrasión lo que lleva a proponer una exposición reciente o bien reiterados ciclos de exposición de escasa duración; 4) todos los materiales líticos tienen origen antrópico y la fracción de menor tamaño $(\leq 5 \mathrm{~mm})$ representa una litificación no intencional del paisaje a microescala; 5 ) las dataciones por OSL indican que los conjuntos arqueológicos estudiados se encuentran sobre depósitos del Pleistoceno tardío final por lo que pueden corresponder a cualquier momento del presente interglaciar.

\section{Agradecimientos}

Esta investigación fue realizada enteramente con fondos propios por lo que la ayuda y asesoramiento de muchos profesionales que desinteresadamente brindaron sus conocimientos, trabajo y experiencia ha sido fundamental para poder llevarla a cabo. Entre ellos no puedo dejar de mencionar especialmente a la Dra. Karen Borrazzo, el Dr. Guillermo Heider, el Dr. Cristian Favier Dubois, el Lic. Roberto Bracco, la Dra. Paula Vitale, el Dr. Gustavo Politis y el equipo que integra a la Unidad Ejecutora INCUAPA-CONICET-UNICEN. También quiero agradecer al Dr. Luis Borrero y al Dr. Roberto Kokot, jurados designados para la defensa, por sus importantes comentarios y aportes. Al gobierno de la provincia de San Luis por su apoyo.

\section{Referencias citadas}

» Balfet, H., Fauvet-Berthelot, M. F. y Monzón, S. (1992). Normas para la descripción de vasijas cerámicas. México D. F.: Centre D’Études Mexicaines et Centroaméricaines (CEMCA).

» Behrensmeyer, A. K. (1978). Taphonomic and ecologic information from bone weathering. Paleobiology, 4(2), 150-162.

"Borrazzo, K. B. (2006). Tafonomía lítica en dunas: una propuesta para el análisis de los artefactos líticos. Intersecciones en Antropología, 7, 247-261.

"Butzer, K. W. 1989. Arqueología - Una Ecología del Hombre. Barcelona: Ediciones Bellaterra.

» Colman, S. M., Pierce, K. L. y Birkeland, P. W. (1987). Suggested terminology for Quaternary dating methods. Quaternary Research, 28, 314-319.

" Fanning, P. C., Holdaway, S. J., Rhodes, E. J. y Bryant, T. G. (2009). The surface archaeological record in arid Australia: geomorphic controls on preservation, exposure and visibility. Geoarchaeology: An International Journal, 24(2), 121-146.

" Favier Dubois, C. M. (2009). Geoarqueología: explorando propiedades espaciales y temporales del registro arqueológico. En R. Barberena, K. Borrazzo y L. A. Borrero (Eds.), Perspectivas actuales en arqueología Argentina (pp. 35-54). Buenos Aires: Editorial Dunken.

"Fernández Jalvo, Y. y Andrews, P. (2016). Atlas of taphonomic identifications. 1001+ images of fossil and recent mammal bone modification. NuevaYork: Springer.

» Forman, S. L., Tripaldi, A. y Ciccioli, P. L. (2014). Eolian san sheet deposition in the San Luis palaeodune field, western Argentina as an indicator of semi-arid environment through the Holocene. Palaeogeography, Palaeoclimatology, Palaeoecology, 411, 122-135.

» Greslebin, H. (1924). Fisiografía y noticia preliminar sobre la arqueología de la región de Sayape (Provincia de San Luis). Buenos Aires: Talleres Gráficos Ferrari Hnos. 
» Greslebin, H. (1928). La antigüedad del hombre en la región de Sayape, Provincia de San Luis, República Argentina (Nota preliminar). En XXIII International Congress of Americanists, (pp. 305-312). Nueva York.

» Heider, G. (2015). Los pueblos originarios en el norte de Pampa Seca. Una mirada arqueológica a los cazadores recolectores del sur de las provincias de Córdobay San Luis, Argentina. (Tesis de Doctorado inédita), Universidad Nacional de Córdoba, Argentina.

» Iriondo, M.yKröhling, D. (1995). El sistema eólico pampeano. Comunicaciones del Museo Provincial de Ciencias Naturales "Florentino Ameghino" (Nueva Serie), 5(1), 5-45.
» Ozán, I. L. y Berón, M. A. (2016). Procesos postdepositacionales del registro cerámico de cazadores recolectores de la provincia de La Pampa. Comechingonia, 20(2): 215-242.

»Schiffer, M. (1987). Formation processes of the archaeological record. Albuquerque: University of New Mexico Press.

»Waters, R. M. (1992). Principles of geoarchaeology. Tucson: The University of Arizona Press.

»Zárate, M. y Tripaldi, A. (2012). The aeolian system of central Argentina. Aeolian Research, 3, 401-417. 